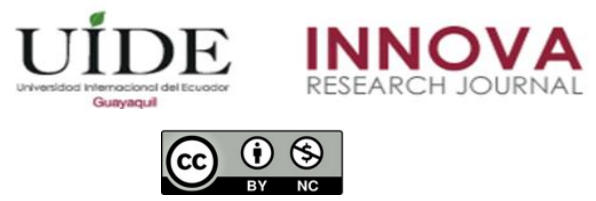

INNOVA Research Journal, ISSN 2477-9024

(Septiembre-Diciembre 2020). Vol. 5, No.3.2 pp. 163-185

DOI: https://doi.org/10.33890/innova.v5.n3.2.2020.1628

URL: http://revistas.uide.edu.ec/index.php/innova/index

Correo: innova@uide.edu.ec

\title{
Quick wins en las organizaciones: una revisión sistemática
}

\section{Quick wins in organizations: a systematic review}

\author{
Marcos Antonio Espinoza Mina \\ (D) https://orcid.org/0000-0003-1530-7243 \\ Universidad ECOTEC, Ecuador
}

Autor para correspondencia: mespinoza@ecotec.edu.ec

Fecha de recepción: 05 de noviembre de 2020 - Fecha de aceptación: 20 de diciembre de 2020

\begin{abstract}
Resumen
Las quick wins son los resultados inmediatos que necesita la organización en la ejecución de proyectos; ya que, son aquella mejora visible que se puede desarrollar rápidamente y con un beneficio inmediato; su impacto no es a largo plazo, pero es vista por todos como algo importante para la organización. Se trabajan con pocos recursos y no deberían darse en un tiempo mayor a 60 o 90 días. Se presenta una revisión sistemática en la que se indica que Springer fue la base de datos que retornó más títulos relacionados; se evidencia que Estados Unidos fue el país desde donde se plantearon más estudios y el año 2017 en el que se presentaron mayores resultados. Se destaca esta estrategia en las áreas de productividad, salud y tecnologías de la información, y se requiere de una adecuada destreza de los gestores para hacer uso de ciertas alertas ya planteadas por experiencias, para no caer en las denominadas "fallas rápidas".
\end{abstract}

Palabras claves: ganancias rápidas; proyectos; estrategias; producción; tecnología.

\begin{abstract}
Quick wins are the immediate results that the organization needs in the execution of projects; since, they are that visible improvement that can be developed quickly and with immediate benefit; its impact is not long-term, but it is seen by everyone as important to the organization. They work with few resources and should not be given in a time greater than 60 or 90 days. A systematic review is presented in which it is indicated that Springer was the database that returned the most related titles; It is evident that the United States was the country from which more studies were proposed and the year 2017 in which the greatest results were presented. This strategy stands out in the areas of productivity, health and information technology, and it requires an adequate dexterity of managers to make use of certain alerts already raised by experiences, so as not to fall into the so-called "rapid failures".
\end{abstract}

Keywords: Quick wins; projects; strategies; production; technology. 


\section{Introducción}

Para adaptarse a los cambios en los climas empresariales, los líderes necesitan desarrollar estrategias múltiples, deben racionalizar las compensaciones entre tiempo, economía y respuesta competitiva. Algunas estrategias podrían ser lentas y acumulativas, mientras que otras son quick wins (QW) que tienen un tiempo de respuesta corto (Wray, 2016).

Se denomina QW, a aquella mejora visible que se puede desarrollar rápidamente y con un beneficio inmediato; su impacto no es a largo plazo, pero es vista por todos como algo importante para la organización. Las QW se trabajan con pocos recursos, son fáciles y rápidas de implementar, no deberían darse en un tiempo mayor a 60 o 90 días; deben culminar con una mejora y brindar un alto nivel de confianza al equipo de trabajo.

Si se planifica un proyecto empresarial que incluye mucho trabajo y un largo tiempo, el recurso humano puede terminar afligido; ellos trabajarán de forma constante, sin que se pueda dar un reconocimiento a alguna parte del trabajo ya realizado; integrar QW en un proyecto puede hacer la diferencia; no hay nada mejor que marcar como cumplido a un entregable de la lista de tareas de un proyecto y esperar poder informarlo en la siguiente reunión de actualización del estado; la energía positiva generada a partir del logro, crea una sensación de satisfacción al equipo que les permite avanzar a la siguiente fase.

Cuando se trata de la mejora continua de procesos, lo que importa es la acción; no importa la capacitación que se brinde, el eslogan que se use o los afiches que se publiquen, si el equipo no se pone en acción de inmediato para hacer las cosas, medirlas y estabilizarlas para que la solución se mantenga. QW es una herramienta poderosa para llevar a los equipos a la acción (Conway-King y Julien, 2018). El equipo de trabajo al ver que los resultados positivos están directamente relacionados con la actividad principal a desarrollar, genera un impulso para colaborar más con dicha actividad.

Muchas QW sirven para impulsar actividades más grandes o proyectos de mediano y largo plazo, a través de la confianza que puede crear. Las QW deben estar casi garantizadas para tener éxito y son celebradas por el equipo de trabajo, ver resultados rápidos después de un esfuerzo es muy motivador. A la vez, estas historias de éxito ocupan un importante espacio de comentarios entre los colaboradores, en lugar de las cosas negativas de la organización.

Es significativo identificar oportunidades para obtener una mejora de rendimiento de forma rápida y tangible; las mejores oportunidades son problemas que se pueden resolver de manera razonablemente rápido, con gastos modestos y que generarán ganancias operativas y financieras visibles (Watkins, 2003). En un proyecto, se pueden descubrir durante el análisis de procesos o la definición de requerimientos. Puede contemplar el rediseño de un formulario, el cuestionar un procedimiento e introducir la eficiencia a través de pequeños cambios, la reducción de algún costo operativo, entre otros objetivos.

Un ejemplo de cómo identificar las QW en diferentes ámbitos es en el área de las tecnologías de información (TI). Sin los servicios de TI, muchas organizaciones no podrían ofrecer sus productos y servicios en el mercado actual. A medida que aumenta la confianza en 
los servicios de TI, también aumentan las expectativas de disponibilidad, confiabilidad y estabilidad; pero estos pueden verse transgredidos. Uno de los procesos más fáciles de implementar que puede tener algunas QW es la gestión de problemas en TI. La simple compilación de una lista de 10 principales incidentes mensuales brindará oportunidades para identificar incidentes recurrentes que le cuestan a la organización debido al tiempo de inactividad, el esfuerzo por restaurar el servicio y la satisfacción del cliente (Case, 2007).

Adicionalmente, es importante señalar que la medida del éxito de una QW no es el tamaño o la velocidad de su impacto en el resultado final, es la sabiduría adquirida en el proceso; si este aprendizaje se cumplió el líder puede trasladar a su equipo al territorio donde las victorias son más difíciles y demoran más (Van Buren y Safferstone, 2009).

En el presente trabajo de investigación se exponen los resultados de una revisión sistemática realizada con el objetivo de conocer las características de las publicaciones relacionadas a la correcta aplicación de QW. Se plantean los siguientes contenidos: se describen la metodología y el protocolo ejecutado; luego se detallan los resultados de la revisión; a continuación, se presenta la discusión de los resultados; y finalmente se plantean las conclusiones y las referencias bibliográficas

\section{Metodología}

La presente investigación es una revisión sistemática, lo cual la convierte en un artículo de síntesis de la evidencia disponible, en el que se realiza una revisión de aspectos cuantitativos y cualitativos de estudios primarios, con el objetivo de resumir la información existente respecto de un tema en particular (Manterola, Astudillo, Arias, y Claros, 2013).

La revisión se realizó siguiendo el protocolo de Ferreras-Fernández, Martín-Rodero, García-Peñalvo, y Merlo-Vega (2016), quienes consideran los siguientes puntos a resolver: (1) definición de las preguntas de revisión, (2) definición de los criterios para la inclusión y exclusión de los estudios, (3) especificación de la estrategia de búsqueda para la identificación de la literatura, (4) revisión del proceso y evaluación de los estudios, (5) extracción de datos, (6) síntesis, análisis y presentación de datos.

\section{Definición de las preguntas de revisión}

El objetivo de la presente revisión sistemática es conocer las características más destacadas de las publicaciones relacionadas a la aplicación de QW en diferentes áreas; para alcanzar lo mencionado se definieron las siguientes preguntas de revisión:

P1. ¿En qué bases de datos, países y años han sido publicados artículos de QW? P2. ¿A qué áreas de aplicación han sido dirigidos los trabajos de investigación relacionados con QW?

P3. ¿Qué consideraciones deben tomarse al momento de aplicar estrategias QW? 


\section{Definición de los criterios para la inclusión y exclusión de los estudios}

\section{Criterios de inclusión:}

Estudios que permiten dar respuesta a la P1, P2 y P3 a través de un proceso de lectura de sus títulos, resúmenes e introducción de los artículos evaluados.

Los trabajos pueden enmarcarse en cualquier área de conocimiento que considere cuestiones relacionadas con las preguntas de revisión.

Son considerados los siguientes tipos de estudio: artículos, documentos de congresos y conferencias.

Se evalúan solo los artículos extraídos de las bases científicas seleccionadas.

Los artículos deben ser escritos en idioma inglés, por ser una lengua internacional con cualidad idónea para la comunicación científica.

\section{Criterios de exclusión:}

Trabajos que no tienen correspondencia con las preguntas de revisión planteadas. Trabajos desarrollados en diapositivas.

Literatura gris.

Los autores plantearon que ante el caso de que los resultados de búsqueda en las bases científicas seleccionadas sean pocas y no den respuesta a todas las preguntas de revisión, se evaluarían otras fuentes de extracción y se incluirían libros; dichas referencias se las trataría de forma separada.

\section{Especificación de la estrategia de búsqueda para la identificación de la literatura}

Se utilizaron las bases de datos científicas y académicas: Springer,Taylor y Francis Online, Wiley Online Library y National Center for Biotechnology Information. Dichas fuentes de extracción fueron elegidas por los autores al realizar una preevaluación de los resultados de búsquedas relacionadas al tema en bases de datos reconocidas por sus buenos contenidos.

Se evaluaron varias palabras clave para formular la cadena de búsqueda y se consideró el término "quick win". El estudio se inició en mayo de 2019 y se decidió buscar publicaciones entre el año 2011 y 2019. Se añadió a la cadena de búsqueda los criterios de inclusión.

Fueron pocos los resultados de la búsqueda en las bases de datos seleccionadas, aplicados los criterios de inclusión y exclusión, razón por la cual los autores decidieron ampliar las fuentes de información y utilizar al motor de búsqueda de Google Académico, se buscaron documentos científicos y técnicos incluidos libros con el término acordado de "quick win", y se amplió el período de tiempo de publicación, desde el 2000 al 2019, la consulta fue presentada por orden de relevancia del mismo motor de búsqueda y sin incluir patentes y citas. A los documentos presentados por el motor de búsqueda se aplicaron los criterios de inclusión y exclusión ya planteados, que permitieron dar respuesta a P1, P2 y P3. 


\section{Revisión del proceso y evaluación de los estudios}

Se tomaron los primeros doscientos resultados de la búsqueda de cada base de datos, se responsabilizó la calidad de los mismos a la evaluación automatizada que realizan las plataformas informáticas de las fuentes bibliográficas de donde se los tomó, ya que dichos motores de búsqueda generan sus resultados por orden de relevancia. Luego de ello, se realizó una primera selección a través de los títulos y resúmenes extraídos.

El grado de acuerdo entre ambos revisores fue alto, lo cual permitió que no se genere ninguna discrepancia, y tenga que consultarse a un tercer investigador. Para gestionar de mejor forma los resultados extraídos se utilizó el administrador de referencias Zotero.

\section{Extracción de datos}

Una vez extraídos y seleccionados en el primer filtro aplicado, se leyó y revisó la literatura en su totalidad para verificar el cumplimiento de los criterios de inclusión. Para la selección de la información más relevante de cada uno de los trabajos, los revisores consideraron de forma permanente el objetivo y las preguntas de investigación. La extracción de información relevante de los estudios primarios, fue ubicada en un formulario elaborado en Microsoft Excel en donde cada revisor completaba y clasificaba los datos obtenidos, para que a partir de ahí pueda ser organizada y explotada la información.

\section{Síntesis, análisis y presentación de datos}

Se tabularon aquellos resultados del formulario levantado, confirmándose los requisitos de inclusión, se clasificaron los trabajos de acuerdo a los descriptores relacionados a P1, P2 y P3.

Se confirmó la calidad de los trabajos de acuerdo a los objetivos y las metodologías documentadas, se identificaron una vez más los contenidos más importantes para lograr relacionarlos en conjunto y encadenarlos para su respectiva presentación.

\section{Resultados}

De las bases de datos definidas fueron seleccionados 18 artículos, es decir aquellos que cumplieron con los criterios de inclusión, no evidenciaron criterios de exclusión y su aporte era significativo. Sin embargo, tal como se señaló en los criterios de inclusión y exclusión definidos, al constatarse que los resultados eran pocos y no daban respuesta en su totalidad a las preguntas de investigación, principalmente a P3, se evaluaron otras fuentes y se incluyó a los libros relacionados, se aplicó la estrategia de búsqueda para la identificación de la literatura definida.

La tabla 1 muestra los estudios elegidos finalmente en este trabajo, que sumaron en su totalidad 24, así como un resumen de sus características, están agrupados por el origen de la extracción y su orden no determina su importancia; los últimos 6 trabajos son aquellos adicionales seleccionados. 


\section{Tabla 1}

Artículos, origen de extracción, área de aplicación y país a donde se dirigió estudio

\begin{tabular}{|c|c|c|c|c|}
\hline Título & Autor & $\mathbf{A}$ & $\mathbf{B}$ & País \\
\hline $\begin{array}{l}\text { Overcoming internal barriers to industrial energy } \\
\text { efficiency through energy audit: A case study of a } \\
\text { large manufacturing company in the home appliances } \\
\text { industry. }\end{array}$ & $\begin{array}{l}\text { Chiaroni, D., Chiesa, V., } \\
\text { Franzò, S., Frattini, F., \& } \\
\text { Manfredi Latilla, V. (2017) }\end{array}$ & 1 & IN & Italia \\
\hline $\begin{array}{l}\text { How the US Federal Communications Commission } \\
\text { Managed the Process of IT Modernization. }\end{array}$ & $\begin{array}{l}\text { Desouza, K. C., Denford, } \\
\text { J. S., \& Krishnamurthy, R. } \\
\text { (2019) }\end{array}$ & 1 & TI & $\begin{array}{l}\text { Estados } \\
\text { Unidos }\end{array}$ \\
\hline $\begin{array}{l}\text { Lean, a Tool Set or a Mind Set? A Healthcare Case } \\
\text { Study. }\end{array}$ & $\begin{array}{l}\text { Guimarães, C. M., \& de } \\
\text { Carvalho, J. C. (2012) }\end{array}$ & 1 & SA & Portugal \\
\hline $\begin{array}{l}\text { Governance in the Technology Era: Implications of } \\
\text { Actor Network Theory for Social Empowerment in } \\
\text { South Asia. }\end{array}$ & $\begin{array}{l}\text { Haque, A., \& Mantode, K. } \\
\text { L. (2013). }\end{array}$ & 1 & PR & $\begin{array}{l}\text { Asia del } \\
\text { Sur }\end{array}$ \\
\hline Youth Entrepreneurship in an Islamic Context. & $\begin{array}{l}\text { Rixon, A., Maritz, A., \& } \\
\text { Fisher, R. (2017). }\end{array}$ & 1 & EM & $\begin{array}{l}\text { Emiratos } \\
\text { Árabes } \\
\text { Unidos }\end{array}$ \\
\hline $\begin{array}{l}\text { Implementation of Six Sigma to Reduce Cost of } \\
\text { Quality: A Case Study of Automobile Sector. }\end{array}$ & Surange, V. G. (2015). & 1 & PR & India \\
\hline $\begin{array}{l}\text { Assessing the impact of energy management } \\
\text { initiatives on the energy usage during the } \\
\text { construction phase of an educational building project } \\
\text { in Ireland. }\end{array}$ & $\begin{array}{l}\text { Gottsche, J., Kelly, M., \& } \\
\text { Taggart, M. (2016) }\end{array}$ & 2 & IN & Irlanda \\
\hline $\begin{array}{l}\text { Sustaining lean in SMEs: Key findings from a 10- } \\
\text { year study involving New Zealand manufacturers. }\end{array}$ & $\begin{array}{l}\text { Grigg, N. P., Goodyer, J. } \\
\text { E., \& Frater, T. G. (2018) }\end{array}$ & 2 & PR & $\begin{array}{l}\text { Nueva } \\
\text { Zelanda }\end{array}$ \\
\hline $\begin{array}{l}\text { A Delphi study. Total Quality Management \& } \\
\text { Business Excellence, }\end{array}$ & $\begin{array}{l}\text { Huang, S.-J., Wu, M.-S., } \\
\text { \& Chen, L.-W. (2013). }\end{array}$ & 2 & TI & China \\
\hline $\begin{array}{l}\text { Planning for school turnaround in the United States: } \\
\text { An analysis of the quality of principal-developed } \\
\text { quick wins. }\end{array}$ & $\begin{array}{l}\text { Meyers, C. V., \& Hitt, D. } \\
\text { H. (2018). }\end{array}$ & 2 & ED & $\begin{array}{l}\text { Estados } \\
\text { Unidos }\end{array}$ \\
\hline $\begin{array}{l}\text { Moving slowly, not breaking enough: Trump's } \\
\text { cybersecurity accomplishments. }\end{array}$ & $\begin{array}{l}\text { Weber, S., \& Cooper, B. } \\
\text { (2017). }\end{array}$ & 2 & TI & $\begin{array}{l}\text { Estados } \\
\text { Unidos }\end{array}$ \\
\hline Making Technology Investments Profitable. & Keen, J. M. (2011) & 3 & TI & $\begin{array}{l}\text { Estados } \\
\text { Unidos }\end{array}$ \\
\hline Designing Metrics and Key Performance Indicators & $\begin{array}{l}\text { Rasmussen, N., Chen, C. } \\
\text { Y., \& Bansal, M. (Eds.). } \\
\text { (2015). }\end{array}$ & 3 & PR & $\begin{array}{l}\text { Estados } \\
\text { Unidos }\end{array}$ \\
\hline Supply Chain Transformation. & Sherman, R. (2013). & 3 & PR & $\begin{array}{l}\text { Estados } \\
\text { Unidos }\end{array}$ \\
\hline $\begin{array}{l}\text { Assessing quick wins to protect critical urban } \\
\text { infrastructure from floods: A case study in Bangkok, } \\
\text { Thailand: Quick wins to protect critical urban } \\
\text { infrastructure. }\end{array}$ & $\begin{array}{l}\text { Zevenbergen, C., van } \\
\text { Herk, S., Escarameia, M., } \\
\text { Gersonius, B., Serre, D., } \\
\text { Walliman, N., ... de Graaf, } \\
\text { R. (2018). }\end{array}$ & 3 & IN & Tailandia \\
\hline $\begin{array}{l}\text { An analysis of policy success and failure in formal } \\
\text { evaluations of Australia's national mental health } \\
\text { strategy (1992-2012). }\end{array}$ & $\begin{array}{l}\text { Grace, F. C., Meurk, C. S., } \\
\text { Head, B. W., Hall, W. D., } \\
\text { Harris, M. G., \& Whiteford, } \\
\text { H. A. (2017) }\end{array}$ & 4 & SA & Australia \\
\hline $\begin{array}{l}\text { A Model for Good Governance of Healthcare } \\
\text { Technology Management in the Public Sector: } \\
\text { Learning from Evidence-Informed Policy }\end{array}$ & $\begin{array}{l}\text { Houngbo, P. Th., } \\
\text { Coleman, H. L. S., } \\
\text { Zweekhorst, M., De Cock }\end{array}$ & 4 & SA & Benín \\
\hline
\end{tabular}




\begin{tabular}{|c|c|c|c|c|}
\hline Título & Autor & $\mathbf{A}$ & B & País \\
\hline Development and Implementation in Benin. & $\begin{array}{l}\text { Buning, Tj., Medenou, D., } \\
\& \text { Bunders, J. F. G. (2017) }\end{array}$ & & & \\
\hline $\begin{array}{l}\text { Targeting Outpatient Drug Safety: } \\
\text { Recommendations of the Dutch HARM-Wrestling }\end{array}$ & $\begin{array}{l}\text { Warlé-van Herwaarden, } \\
\text { M. F., Kramers, C., }\end{array}$ & 4 & SA & $\begin{array}{l}\text { Países } \\
\text { bajos }\end{array}$ \\
\hline Task Force. & $\begin{array}{l}\text { Sturkenboom, M. C., van } \\
\text { den Bemt, P. M. L. A., \& } \\
\text { De Smet, P. A. G. M. } \\
\text { (2012). }\end{array}$ & & & \\
\hline $\begin{array}{l}\text { Implementing process-oriented knowledge } \\
\text { management strategies. }\end{array}$ & $\begin{array}{l}\text { Maier, R., \& Remus, U. } \\
\text { (2003). }\end{array}$ & 5 & GE & Alemania \\
\hline $\begin{array}{l}\text { Early detection of maternal deaths in Senegal } \\
\text { through household-based death notification } \\
\text { integrating verbal and social autopsy: A community- } \\
\text { level case study. }\end{array}$ & $\begin{array}{l}\text { Moshabela, M., Sene, M., } \\
\text { Nanne, I., Tankoano, Y., } \\
\text { Schaefer, J., Niang, O., \& } \\
\text { Sachs, S. E. (2015). }\end{array}$ & 6 & SA & Senegal \\
\hline $\begin{array}{l}\text { Assessing the impact of construction waste } \\
\text { reduction on selected projects in Ireland. }\end{array}$ & $\begin{array}{l}\text { Gottsche, J., \& Kelly, M. } \\
\text { (2018) }\end{array}$ & 7 & $\mathrm{ME}$ & Irlanda \\
\hline Can Small Victories Help Win the War. & $\begin{array}{l}\text { Gal, D., \& McShane, B. } \\
\text { B. (2012) }\end{array}$ & 8 & GE & $\begin{array}{l}\text { Estados } \\
\text { Unidos }\end{array}$ \\
\hline Preparing for productivity intervention initiatives. & $\begin{array}{l}\text { Crouzet, B., W. Parker, } \\
\text { D., \& Pathak, R. (2014) }\end{array}$ & 9 & GE & Australia \\
\hline $\begin{array}{l}\text { Prioritizing and optimizing sustainable measures for } \\
\text { food waste prevention and management. }\end{array}$ & $\begin{array}{l}\text { Cristóbal, J., Castellani, } \\
\text { V., Manfredi, S., \& Sala, S. } \\
\text { (2018) }\end{array}$ & 10 & $\mathrm{ME}$ & Italia \\
\hline
\end{tabular}

“A” BASE DE DATOS “1” Springer, "2" Taylor\&Francis Online, "3" Wiley Online Library, "4" National Center for Biotechnology Information, "5" SemanticScholar, "6" BMC Health Services Research, "7" ICE Virtual Library, "8" SAGE journals, "9" Emerald insight, "10" ScienceDirect

"B" ÁREA DE APLICACIÓN, "IN" Ingeniería, "TI" Tecnologías de la información, "SA" Salud, "PR"

Productividad, "EM" Emprendimiento, "ED" Educación, "GE” Gestión, "ME” Medioambiente

Fuente: Elaboración propia

Para dar respuesta a P1, se puede indicar que Springer fue la base de datos de publicaciones científicas que presentó más artículos relacionados con QW. El país desde el cual se realizaron la mayor cantidad de publicaciones fue en Estados Unidos, y los años más representativos para la presentación de resultados de este tipo de investigación fueron 2017 y 2018; ver tablas 2, 3 y 4 .

\section{Tabla 2}

Bases de datos de donde se extrajeron los artículos

\begin{tabular}{lll}
\hline Base de datos & Cantidad & $\%$ \\
\hline Springer & 6 & 25 \\
Taylor\&Francis Online & 5 & 21 \\
Wiley Online Library & 4 & 17 \\
National Center for Biotechnology Information & 3 & 13 \\
Otras & 6 & 25 \\
\hline
\end{tabular}

Fuente: Elaboración propia 


\section{Tabla 3}

País en el que se realizó la publicación

\begin{tabular}{lll}
\hline País de publicación & Cantidad & $\%$ \\
\hline Asia del Sur & 1 & 4 \\
Australia & 2 & 8 \\
Benín & 1 & 4 \\
China & 1 & 4 \\
Emiratos Árabes & 1 & 4 \\
Estados Unidos & 7 & 29 \\
India & 1 & 4 \\
Irlanda & 2 & 8 \\
Italia & 2 & 8 \\
Nueva Zelanda & 1 & 4 \\
Países Bajos & 1 & 4 \\
Portugal & 1 & 4 \\
Tailandia & 1 & 4 \\
Alemania & 1 & 4 \\
Senegal & 1 & 4 \\
\hline
\end{tabular}

Fuente: Elaboración propia

\section{Tabla 4}

Año de publicación de los artículos revisados

\begin{tabular}{cll}
\hline $\begin{array}{c}\text { Año de } \\
\text { publicación }\end{array}$ & Cantidad & $\%$ \\
\hline 2003 & 1 & 4 \\
2011 & 1 & 4 \\
2012 & 3 & 13 \\
2013 & 3 & 13 \\
2014 & 1 & 4 \\
2015 & 3 & 13 \\
2016 & 1 & 4 \\
2017 & 5 & 21 \\
2018 & 5 & 21 \\
2019 & 1 & 4 \\
\hline & 24 & 100 \\
\hline
\end{tabular}

Fuente: Elaboración propia 
Dando respuesta a P2, se puede señalar que los artículos revisados también muestran las áreas de aplicación a las que dirigieron los estudios, siendo las más representativas las de productividad, salud y TI; ver tabla 5.

\section{Tabla 5}

Áreas de aplicación a los que se dirigieron los estudios revisados.

\begin{tabular}{lll}
\hline Área de aplicación & Cantidad & $\%$ \\
\hline Ingeniería & 3 & 13 \\
TI & 4 & 17 \\
Salud & 5 & 21 \\
Productividad & 5 & 21 \\
Emprendimiento & 1 & 4 \\
Educación & 1 & 4 \\
Gestión & 3 & 13 \\
Medioambiente & 2 & 8 \\
\hline & 24 & 100 \\
\hline
\end{tabular}

Fuente: Elaboración propia

Con referencia a P3, los aportes más significativos lo dieron los siguientes autores: Maier y Remus (2003), Watkins (2003), Corporate Executive Board (2006), Van Buren y Safferstone, (2009), Keen (2011), Gal y McShane (2012), Rollin (2012), Crouzet, W. Parker, y Pathak, (2014), Wray (2016), Conway-King y Julien (2018), Meyers y Hitt (2018).

\section{Discusión}

\section{Sobre las bases de datos, países y años han sido publicados artículos de QW (P1)}

Aunque Springer ocupa el primer lugar en el número de artículos relacionados con QW, encontrados en el presente trabajo y aplicando los criterios de inclusión y exclusión, no se puede dejar de considerar notariedad a Taylor y Francis Online y las otras dos bases de datos consideradas inicialmente, debido a los pocos artículos finalmente seleccionados.

Es contundente que Estados Unidos es el país en el que más estudios a tratado con las QW y en el último par de años nuevamente algunos especialistas vuelven a considerar el estudio de la aplicación de las QW como una herramienta estratégica en las organizaciones.

\section{Sobre la aplicación de QW (P2)}

\section{En el área de productividad}

Haque y Mantode (2013) estudiaron el proyecto Village Knowledge Center (VKC) en India y el proyecto Acceso a la Información (A2I) en Bangladesh, los cuales proporcionaron 
pruebas sólidas de cómo el desarrollo social dirigido por las TI y comunicación puede ser eficaz a corto plazo. El estudio reveló que, en lugar de introducir un gran cambio a través de un gran proyecto, un enfoque gradual e incremental puede tener un impacto más amplio y más significativo en la sociedad. Esto se debe a que, al mantener las cosas simples, la definición estándar se puede replicar fácil y rápidamente para servir a un mayor número de personas en una gran variedad de formas pequeñas. Por ejemplo, en los proyectos QW para A2I, los agricultores habían obtenido información valiosa rápidamente a un costo insignificante.

El costo de calidad no es el precio de crear un producto o servicio de calidad, es el costo de no crear un producto o servicio de calidad. Surange (2015) presentó un estudio de caso, que permitió la aplicación de la metodología Six Sigma, que ayuda a reducir los costos, en una amplia gama de actividades de fabricación; planteó seis fases consecutivas, siendo una de ellas la fase de definición, y sus principales actividades son: identificar los proyectos que están vinculados a la gran objetivo que mejoren el margen de rendimiento en un 10\%; seleccionar el equipo que está listo para colaborar; preparar el carácter del equipo y conseguirlo aprobado; entrenar a los miembros del equipo; mapear el proceso; realizar análisis de valor; recorrer el proceso; analizar los datos pasados desde varios puntos de vista; identificar QW e implementar; finalmente registrar resultados de QW.

Los KPI (Key Performance Indicator) se definen como métricas que se utilizan para ayudar a una organización a definir y medir el progreso de actividades, permitiendo cumplir con los objetivos de la organización. Rasmussen, Bansal, y Chen (2009), exponen los diez pasos que se recomiendan utilizar en la planificación de un proyecto de diseño de KPI. En el paso tres se debe decir qué categoría de panel de control es la más importante y en qué orden se deben diseñar. Se recomienda construir un tablero de mandos de alta visibilidad por primera vez para obtener una QW, ya que motivará la participación y aumentará el interés en el proyecto del tablero general. El paso cuatro se pide que se decida si la implementación del tablero debe realizarse a nivel departamental / divisional o en la parte superior, y si debería existir un plan para luego continuar desplegando los paneles de manera horizontal a través de las divisiones, o de arriba hacia abajo. El orden en el que se implementa depende de las posibilidades de una QW, el retorno de la inversión esperado, el interés del departamento, la disponibilidad de datos, etc.

Sherman (2013), proporciona una hoja de ruta para ayudar a guiar las estrategias de la cadena de suministro y conducir este proceso a mejorar su rendimiento y hacerlo diferenciado. El autor expone que el mapeo de procesos, la medición, la evaluación comparativa y el análisis, permiten priorizar los proyectos de mejora y la implementación de las mejores prácticas. Recomienda que al comenzar cualquier proceso que potencialmente cierre una brecha de desempeño del inventario o libere capital de trabajo, se debe determinar el riesgo y el rendimiento de cada proyecto posible. Si un proyecto tiene un alto rendimiento y un bajo riesgo, es una oportunidad de QW. Sherman, sugiere seleccionar oportunidades piloto de QW para liberar fondos en el corto plazo y obtener una gestión adicional y apoyo de equipo; recuerda la necesidad de movilizar al equipo para que acompañe en el viaje de las mejoras y las QW son catalizadores para lanzarlos.

Ahora bien, para ayudar a aumentar la productividad del sector manufacturero de Nueva Zelanda (NZ), el gobierno intentó implementar el programa LEAN, que se enfoca en minimizar

Esta obra se comparte bajo la licencia Creative Common Atribución-No Comercial 4.0 International (CC BY-NC 4.0) Revista de la Universidad Internacional del Ecuador. URL: https://www.uide.edu.ec/ 
las pérdidas al mismo tiempo que maximiza la creación de valor, a través de un programa de inversión dirigido. Grigg, Goodyer, y Frater (2018) evaluaron la efectividad y el éxito de esta estrategia, tomando una muestra de fabricantes pequeños y medianos que habían sido parte del programa LEAN del gobierno. Utilizando el modelo "Lean Iceberg Sostenible" como base teórica, desarrollaron un protocolo de estudio de caso basado en construcciones clave para la sostenibilidad de LEAN.

Grigg, Goodyer, y Frater manifiestan que el proceso de implementación LEAN, normalmente debería ser liderado por alguien representativo apoyado por un equipo. La mayoría de las empresas nombraron a un campeón interno de LEAN para supervisar la iniciativa, pero no contrataron al consultor para desarrollar sus capacidades y así liderar los cambios en el futuro, tampoco se preparó se preparó satisfactoriamente mediante capacitación al campeón con las habilidades necesarias. La mayoría de las organizaciones aún dependían en gran medida del consultor para impulsar mejoras y cambios. Los consultores hicieron un buen trabajo con LEAN, pero su tiempo se invirtió en la entrega de QW en lugar del desarrollo de capacidades internas a largo plazo para continuar impulsando LEAN. La tendencia a centrarse en las implementaciones de herramientas y técnicas de QW no crea el cambio cultural necesario para permitir una inclinación sostenible. El estudio refuerza la importancia vital de "estrategia y alineación", "liderazgo" y "comportamiento y compromiso".

\section{En el área de salud}

Houngbo et al. (2017), documentaron el proceso de desarrollo de una estrategia para implementar un modelo de buen gobierno para la gestión de la tecnología de la salud en el sector público, basado en las lecciones aprendidas de veinte años de experiencia en el desarrollo y la implementación de políticas en la República de Benín, un país ubicado en el oeste de África. El modelo comprende seis fases y una de ellas es el desarrollo de instrumentos de políticas para pruebas piloto, basadas en QW para comprender las respuestas del sistema al cambio. Plantean esta estrategia debido a que determinaron que, las condiciones que deben alinearse para alcanzar los objetivos de ciertas intervenciones políticas requieren diferentes combinaciones de recursos, tiempo y factores contingentes. Los autores identificaron instrumentos de políticas de QW que no requerían la presencia de otros factores contingentes.

Además, Grace et al. (2017), en el análisis documental realizado a seis trabajos de evaluación correspondientes a tres planes estratégicos nacionales australianos de salud mental, extrajeron una lista de indudables éxitos y fracasos. Un indudable éxito se definió como la aplicación de una política pública a una iniciativa particular que cumplía con todas las medidas de éxito mediante las cuales se evaluó y no mostró señales de fracaso. Los éxitos indudables se clasificaron además como QW, es decir, los alcanzados dentro de la vida de un solo plan, o éxitos acumulativos, aquellos que abarcan dos o más planes. Los autores identificaron once QW en el transcurso de esos planes estratégicos. Encontraron una asociación inversa entre el grado de complejidad involucrado en la implementación de un objetivo particular y su probabilidad de éxito.

Moshabela et al. (2015), plantean que en un estudio a nivel comunitario en Senegal se reveló un aumento aparente en las muertes maternas, lo que requirió un esfuerzo de colaboración 
de múltiples partes interesadas en remediar esta situación, quienes aprovecharon las QW para aliviar la gravedad, pero también las iniciativas sostenibles a largo plazo. La planificación conjunta entre las autoridades gubernamentales, no gubernamentales y los proveedores, a través de un modelo de cambio rápido de ciclo, permitió la implementación exitosa de QW, que parecen haber limitado el número de muertes maternas asociadas a hospitales en el período posterior de 12 meses.

De acuerdo a Warlé-van Herwaarden, Kramers, Sturkenboom, van den Bemt, y De Smet (2012), dos estudios observacionales holandeses HARM (admisiones hospitalarias relacionadas con medicamentos) e IPCI (información de atención primaria integrada) demostraron que aproximadamente el $5 \%$ de todas las admisiones hospitalarias no planificadas están asociadas con eventos adversos de medicamentos (ADE), de los cuales entre el 40 y $46 \%$ son potencialmente prevenibles. Estos estudios impulsaron el inicio de un grupo de trabajo multidisciplinario para reducir el número de HARM. El estudio planteado tuvo como objetivo identificar las ADE más relevantes y desarrollar un número limitado de recomendaciones para intervenciones concretas, que deberían ser factibles y relativamente fáciles de convertir en alertas computarizadas de seguridad de medicamentos.

Finalmente, se elaboró 34 recomendaciones específicas para reducir los HARM potencialmente prevenibles a través de QW. Muchas de esas recomendaciones ya estaban presentes en las pautas de tratamiento de la práctica clínica prevalecientes, por lo que buscar una rápida reducción de los HARM observados tiene más que ver con implementar y reforzar las pautas existentes que con reemplazarlas. Sin embargo, también se planteó que los debates sobre la mejora de la seguridad de los medicamentos en pacientes ambulatorios no solo deben centrarse en las QW a corto plazo, sino también en las estrategias de reducción de riesgos a más largo plazo.

Por otra parte, con el fin de evaluar qué tan integrados están los principios y herramientas del modelo de gestión LEAN, en la atención médica y cómo las organizaciones sostienen los beneficios, Guimarães y de Carvalho (2012) realizaron un estudio de caso en una organización de atención médica con 21 unidades de diagnóstico que llevan a cabo los eventos de Kaizen (mejora continua). Entre sus señalamientos se encuentra que una cosa es enseñar a las personas las herramientas y técnicas, otra es lograr que las apliquen en sus áreas de trabajo, ya que se requiere una mentalidad de mejora continua que conduzca a la cooperación en el mantenimiento de los primeros logros QW, para que toda la organización pueda jugar afinadamente de la misma manera y no de forma individual.

\section{En el área de TI}

Huang, Wu, y Chen (2013), presentan los resultados de un estudio Delphi modificado, de profesionales de gestión de servicios de TI (ITSM) sobre la importancia relativa de varios factores críticos de éxito para su implementación. En dicho trabajo, se planteó que usar una QW para demostrar la utilidad de la implementación de ITSM es importante, especialmente en relación con el análisis de costo-beneficio. En general, las implementaciones de ITSM son proyectos a largo plazo, con $\mathrm{QW}$, es muy probable que el miembro del equipo retenga fácilmente su energía para la mejora continua y no perder rápidamente su entusiasmo. 
Así mismo, Desouza, Denford, y Krishnamurthy (2019) examinaron cómo la Comisión Federal de Comunicaciones (FCC) de los Estados Unidos ejecutó su esfuerzo de modernización de la TI. En 2013, la FCC gastó alrededor del 80\% de su presupuesto de TI en el mantenimiento de sus sistemas heredados. Reconociendo los ocho años anteriores de implementación fragmentada; el nuevo CIO (Chief Information Officer) realizó un inventario de infraestructura humana y de TI con un enfoque para comprender las vulnerabilidades y el nivel de exposición a la seguridad cibernética. Además, el CIO también tomó medidas para comprender los sentimientos de los empleados, clientes y el liderazgo principal sobre los esfuerzos de modernización de TI. La modernización produjo cinco lecciones, y una de ellas fue que se debe planificar un enfoque por fases que logre QW en la implementación en la nube, que aumente el impulso y reduzca la resistencia a la modernización de los sistemas de TI. Cuanto más grande sea el proyecto, más probabilidades hay de que falle, y el gobierno está lleno de proyectos grandes que tienden a tener un desempeño inferior.

Jack M. Keen (2011), se centró en cómo prevenir, detectar y detener las fugas de valor con el fin de maximizar la realización de valor de las inversiones en TI de todo tipo. El autor plantea que la gestión existe para maximizar el valor, todo lo demás es un medio para ese fin; por lo tanto, es sorprendente que tres cuartas partes de las inversiones habilitadas por TI no entreguen su valor esperado. Las acciones, no las palabras, crean valor. Entre sus múltiples señalamientos enfatiza que para lograrlo se debe comenzar con las QW más importantes que mejor se adapten a la organización.

Weber y Cooper (2017), expusieron que al final del primer año de la administración del presidente Donald Trump, se había hecho relativamente poco, al menos públicamente, para cambiar la política de ciberseguridad y la conversación de estrategia dentro de los Estados Unidos; mientras tanto, el entorno de ciberseguridad se estaba deteriorando visiblemente. Sin embargo, señalaron que hay un montón de espacio para QW en las cosas que importan, y algunas de ellas establecerán el escenario para objetivos más ambiciosos en el futuro. Una QW que plantearon los autores es que el poder ejecutivo desarrolle nuevas formas de incorporar a los tecnólogos más innovadores del sector privado al servicio nacional, al tiempo que permita que ese talento se mantenga conectado a la innovación cibernética del sector privado.

\section{En el área de Ingeniería}

Zevenbergen et al. (2018), presentaron un procedimiento de detección denominado metodología de escaneo rápido, calificado también como QW, que tiene el propósito de brindar orientación a los operadores de redes y tomadores de decisiones sobre la identificación y calificación de las redes de infraestructura crítica y los edificios de puntos críticos, que pueden estar en riesgo de inundación, y evaluar dónde será más factible y rentable la intervención. Los autores desarrollaron talleres y entrevistas con partes interesadas y expertos en ciudades piloto, como Bangkok, París y Dordrecht, para probar y desarrollar el método. El enfoque lo aplicaron para evaluar las QW en tres comunidades urbanas en la Región Metropolitana de Bangkok. La metodología de escaneo rápido demostró proporcionar información práctica y útil con un enfoque en las selecciones más sencillas para reducir la vulnerabilidad e identificar acciones de bajo. 
Según Chiaroni, Chiesa, Franzò, Frattini, y Manfredi Latilla (2017), la eficiencia energética desempeña un papel clave en la reducción del consumo mundial de energía, especialmente en el sector industrial, con un impacto indirecto positivo en la competitividad de las empresas industriales; ellos, presentan y analizan una metodología novedosa para la auditoría de energía desarrollada e implementada por una gran empresa de fabricación activa en la industria de electrodomésticos. Esta auditoría energética fue efectiva para superar las barreras típicas que obstaculizan la adopción de medidas de eficiencia energética (EEM); en las primeras fases del proceso de auditoría energética, se introdujo una corta serie de actividades, entre ellas el establecimiento de criterios para seleccionar EEM que priorizan aquellas iniciativas de eficiencia energética que ofrecen rendimientos rápidos y visibles, es decir para ser utilizados como proyectos de QW que comunican y prueban de manera tangible los beneficios y la viabilidad de EEM.

Gottsche, Kelly, y Taggart (2016) desarrollaron un estudio en el que se investigó si la implementación de medidas de reducción y QW de la energía durante la fase de construcción de un proyecto, tendría un impacto en términos de costo, uso de energía y emisiones de CO2. En sus estudios emplearon la metodología de AR (Action Research), adaptada de Baskerville (1999), que consta de cuatro etapas: diagnóstico, planificación de la acción, toma de acción y evaluación. En la segunda etapa seleccionaron una serie de medidas de bajo coste y de QW para la implementación. Los resultados sugirieron que las iniciativas implementadas tuvieron un impacto significativo que resultó en un ahorro de costos, una reducción en el uso de energía y una reducción de emisiones de $\mathrm{CO} 2$.

\section{En el emprendimiento y la educación}

Rixon, Maritz, y Fisher (2017) conceptualizaron un marco de un sistema nacional de emprendimiento juvenil en los Emiratos Árabes Unidos (EAU), aprovechando los mecanismos de apoyo de los gobiernos y la comunidad para superar la naturaleza individual del emprendimiento. Se plantearon seis recomendaciones basadas en el tema de mejorar el soporte coordinado y se clasificaron en QW (pueden completarse en un año), y en actividades a largo plazo (tienen un horizonte de tiempo de 3 a 5 años); las de QW se plantean y justifican a continuación.

Recopile y distribuya historias de jóvenes emprendedores exitosos. Una de las brechas notables es la falta visible del compromiso de los jóvenes emprendedores. Se debería, identificar un número exitoso de emprendedores e involucrarlos en una serie de foros que podrían grabarse y digitalizarse en video.

Recopile y distribuya historias del conocimiento de padres empresarios. Tanto los jóvenes como las partes interesadas deben participar en actividades en las que los padres o familiares compartan sus aprendizajes y conocimientos sobre negocios y emprendimiento.

Establecer un punto de referencia en el actual emprendimiento juvenil. Antes de hacer una inversión y contribución considerables al tema de emprendimiento, innovación y creatividad, tiene sentido realizar un punto de referencia inicial del emprendimiento juvenil actual, para poder 
ver qué impacto y progreso se va logrando; también podría ayudar a identificar cuáles son los niveles de interés y emprendimiento juvenil y qué sectores / industrias atraen a los jóvenes.

Ahora bien, Meyers y Hitt (2018), evaluaron las QW formales según lo planificado por los directores de escuelas que participaron en un programa de liderazgo de cambio en los Estados Unidos, respaldada por el gobierno federal. Los autores señalaron que los directores de las escuelas que tuvieron la tarea de desarrollar estrategias QW generalmente no tuvieron éxito.

Reflejaron que, en la guía de práctica sobre el cambio, se identificó correctamente la necesidad de señalar el cambio, pero la forma en que ese cambio se planifica (y se realiza) de manera efectiva permanecía sin declarar. En ese sentido, Meyers y Hitt exponen que, aunque una QW es solo una pequeña parte del proceso general de planificación de mejoras, hay implicaciones potencialmente mayores. La falla en la creación de un plan de QW, da como resultado oportunidades perdidas para mostrar que se está produciendo un cambio. Los autores indican que las QW, al menos en la forma planteada al momento de hacer el estudio, carecían de desarrollo estratégico.

\section{En el medio ambiente}

Cristóbal, Castellani, Manfredi, y Sala (2018), desarrollaron una investigación que aborda la situación en la que un decisor tiene que diseñar un programa de prevención de desperdicio de alimentos considerando los recursos económicos limitados para lograr la mayor prevención de impacto ambiental a lo largo de todo el ciclo de vida de los alimentos. Los resultados mostraron que generalmente se sigue el orden establecido en la jerarquía de residuos, además que la inclusión de los criterios económicos, como una restricción presupuestaria, muestra que no siempre las medidas de prevención se priorizan antes de las medidas de reutilización o reciclado, ya que las medidas de reutilización y recuperación de reciclado pueden ser más rentables. Proponen una metodología que utiliza la evaluación del ciclo de vida y la programación matemática, la cual reveló ser especialmente útil en la identificación QW, medidas que siempre deben ser priorizadas, ya que evitan un alto impacto ambiental a bajo costo.

Por otro lado, Gottsche y Kelly (2018) hicieron un estudio que identifica el ahorro de costos y el impacto ambiental reducido, asociado con la implementación de iniciativas de reducción de desechos durante el proceso de construcción de cinco proyectos de salud y educación en Irlanda. Las actividades que realizaron fueron parte de un enfoque propuesto por los autores, enmarcado por el modelo cíclico propuesto por Susman y Evered en 1978, el cual se estructuró por etapas en donde la segunda y la tercera estaban relacionada con la identificación y comunicación de QW. Se implementaron un total de 34 iniciativas de reducción de desperdicios QW, en los cinco sitios de estudio de caso, que incluyeron 27 cuantificadas en términos de costos y ahorro de dióxido de carbono. El estudio finalmente encontró que las medidas de reducción de desechos QW pueden aumentar los márgenes de ganancia y reducir el impacto ambiental.

\section{Consideraciones en las estrategias QW (P3)}

Gal y McShane (2012), hicieron uso de un conjunto de datos obtenidos de una firma de liquidación de deuda y presentaron hallazgos que sugieren que el completar subtareas discretas, 
podría motivar a los consumidores a persistir en la búsqueda de un objetivo. Es decir que cuando se persigue un objetivo a largo plazo las personas deben centrarse en marcar los elementos de su lista de tareas, en lugar de centrarse simplemente en avanzar hacia su objetivo en un sentido absoluto. En la práctica, esto podría implicar centrarse en completar tareas relativamente cortas y simples relacionadas con objetivos antes que en tareas largas y complejas.

Así mismo Wray (2016) señala que, al desarrollar estrategias exitosas, los líderes empresariales necesitan racionalizar las compensaciones entre el tiempo, la economía y la respuesta competitiva. Algunas estrategias pueden ser de movimiento lento y acrecentadas, pero mostrar resultados acumulativos durante períodos prolongados, mientras que otras pueden ser QW que tienen un cambio más corto.

Meyers y Hitt (2018) exponen que quienes dirigen un plan de QW deben de poseer perspectivas y habilidades para crear planes significativos y de calidad, y los proveedores de apoyo y desarrollo deben guiar eficazmente para la creación exitosa de QW. Se explica que la calidad de las QW es un asunto de habilidad o voluntad, o de ambos. Los que dirigen fallan si: (a) no saben cómo diseñar una QW de alta calidad o (b) no entienden completamente las razones para diseñar QW y cómo se vinculan con el esfuerzo de cambio.

Por otra parte, Rollin (2012) plantea la interrogante ¿Cómo podría estar mal algo con una QW? A la que responde señalando que los nuevos líderes a menudo carecen de perspectiva y eligen el objetivo equivocado, disparan a las victorias en lugar de ayudar al departamento a hacer la transición a un nuevo liderazgo y, a menudo, dañan a las personas y el proceso. Las QW que elijan los líderes les expresará a los demás miembros del equipo lo que les importa a ellos, por eso deben elegir QW inteligentes.

Crouzet, W. Parker, y Pathak (2014), señalan que la gestión y la superación de la oposición al cambio comienzan por evaluar los tipos de resistencia; y esta clasificación formará la base para el desarrollo de una estrategia de implementación para reducirla, que incluye crear, preparar y urgencia para el cambio, crear una visión para el cambio, hacer que los empleados participen en el esfuerzo de cambio, capacitar y capacitar a los empleados, comunicar eficazmente el cambio, crear y planificar QW y volver a congelar la organización.

Con lo anteriormente expuesto, en los siguientes dos subtítulos, se plantean algunas consideraciones, las cuales permitirán reforzar el conocimiento de las estrategias QW y las advertencias en su implementación.

\section{El nuevo líder en la empresa y las QW}

Corporate Executive Board (2006) en su guía para ayudar a los nuevos gerentes de primera línea a hacer una transición exitosa a la administración, recomienda romper el plan de transición en trozos manejables. Al dividir el plan en partes de 30 días, simplificará las cosas y creará un camino claro para lograr QW. Se sugiere que más allá de definir los objetivos de trabajo individuales, un buen plan de transición le permitirá aprovechar la red de soporte; se debe asegurar que el plan cumpla con lo siguiente: 
Describe los pasos para una coordinación clara con el gerente anterior.

Identifica a los compañeros críticos para saber y aprender.

Crea una base para establecer credibilidad (y lograr "QW" con sus informes directos).

Establece las bases para una relación saludable con el nuevo gerente.

Watkins (2003) plantea que, en una nueva posición de liderazgo, no se conoce exactamente los desafíos que se avecinan, ni cómo se los podría enfrentar; todo lo que sabe es que se cuenta con los primeros tres meses para estar en la cima del trabajo y avanzar, o fallar. El nuevo líder, luego de la comprensión de las prioridades y los objetivos para el cambio de comportamiento, puede proceder a crear planes detallados sobre cómo garantizar las QW, y se recomienda hacerlo en dos fases, la primera que permita crear credibilidad en los primeros 30 días (ver tabla 6) y la segunda para decidir dónde concentrará la energía para lograr mejoras tempranas de rendimiento en los siguientes 60 días.

\section{Tabla 6}

\section{QW para construir credibilidad personal}

\begin{tabular}{|c|c|}
\hline Acción & Detalles a accionar \\
\hline $\begin{array}{l}\text { Pensar en cómo se } \\
\text { "conectará" el nuevo líder con } \\
\text { su organización }\end{array}$ & $\begin{array}{l}\text { ¿Qué mensajes se quiere transmitir sobre quién es el nuevo } \\
\text { líder y qué representa? ¿Cuáles son las mejores maneras de } \\
\text { transmitir esos mensajes? }\end{array}$ \\
\hline $\begin{array}{l}\text { Identificar las audiencias } \\
\text { clave y crear mensajes } \\
\text { personalizados para cada uno }\end{array}$ & $\begin{array}{l}\text { Los mensajes no tienen que ser sobre lo que se planea } \\
\text { hacer. Deberían centrarse en quién es el nuevo líder, los } \\
\text { valores y objetivos que representa, el estilo y la forma en que } \\
\text { se planea hacer negocios. }\end{array}$ \\
\hline $\begin{array}{l}\text { Pensar en los compromisos } \\
\text { formales del proceso }\end{array}$ & $\begin{array}{l}\text { ¿Cómo se presentará el nuevo líder? ¿Las primeras } \\
\text { reuniones con informes directos deben ser individuales o en } \\
\text { grupo? ¿Estas reuniones serán informales o se centrarán de } \\
\text { inmediato en temas de negocios y evaluaciones? ¿Qué otros } \\
\text { canales, como el correo electrónico y el video, se utilizará } \\
\text { para presentar al nuevo líder más ampliamente? ¿Tendrá } \\
\text { reuniones anticipadas en otros lugares donde su organización } \\
\text { tenga instalaciones? }\end{array}$ \\
\hline $\begin{array}{l}\text { Identificar y actuar lo más } \\
\text { rápido posible para eliminar } \\
\text { los procesos irritantes menores } \\
\text { pero persistentes en su nueva } \\
\text { organización. }\end{array}$ & $\begin{array}{l}\text { Identificar las relaciones externas tensas y comenzar a } \\
\text { repararlas. Eliminar las reuniones redundantes, acortar las } \\
\text { reuniones excesivamente largas. Mejorar los problemas del } \\
\text { espacio físico. }\end{array}$ \\
\hline
\end{tabular}

Fuente: Elaboración propia basado en Watkins (2003)

Watkins también señala que desarrollar credibilidad personal y algunas relaciones clave ayudan a obtener algunas QW. Sin embargo, también debe identificar oportunidades para obtener una mejora rápida y tangible del rendimiento en el negocio, para ello debe: mantener las metas a largo plazo en la mente, identificar algunos puntos focales prometedores, concentrarse en dichos 
puntos, lanzar proyectos piloto, elevar agentes de cambio y aprovechar los proyectos piloto para introducir nuevos comportamientos.

Por otra parte, de las más de treinta acciones QW analizadas por Keen (2011) se recomienda cuatro, identificadas como aspectos esenciales en cualquier estrategia de mejora de valor: hacerla vocal y visible, poner temas de valor en las agendas de reuniones de los líderes, hacer una auditoría rápida al caso de negocio y presentar cómo el valor oculto puede ser descubierto. De igual manera Keen señala que todas las QW que se puedan describir de una compañía se adaptarán necesariamente a todas las empresas o culturas. En la tabla 7 se proporciona una orientación de prácticas de QW generales, en función de la "necesidad de valor" de la organización.

\section{Tabla 7}

Mejores prácticas para aumentar el valor por tipo de escenario

\begin{tabular}{|c|c|c|}
\hline Valor Necesario & Ejemplos de metas & Ejemplos de buenas prácticas \\
\hline $\begin{array}{l}\text { Mejorar el } \\
\text { compromiso de las } \\
\text { partes interesadas }\end{array}$ & $\begin{array}{l}\text { - Motivar a los interesados } \\
\text { para que estén más } \\
\text { centrados en el valor. } \\
\text { - Acelerar y fortalecer la } \\
\text { participación de los } \\
\text { principales interesados }\end{array}$ & $\begin{array}{l}\text { - Aproveche el patrocinador ejecutivo } \\
\text { comprometido } \\
\text { - Implique profundamente a grupos de } \\
\text { partes interesadas clave } \\
\text { - Obtenga el apoyo visible de la alta } \\
\text { dirección }\end{array}$ \\
\hline $\begin{array}{l}\text { Construir mejores } \\
\text { casos de negocios }\end{array}$ & $\begin{array}{l}\text { - Encontrar valor oculto } \\
\text { - Convertir intangibles en } \\
\text { tangibles cuantificados. } \\
\text { - Capturar componentes de } \\
\text { valor financiero y no } \\
\text { financiero. } \\
\text { - Evitar el conteo doble }\end{array}$ & $\begin{array}{l}\text { - Defina claramente el alcance y la } \\
\text { audiencia } \\
\text { - Descubra valor oculto } \\
\text { - Utilice un marco para el flujo de } \\
\text { efectivo libre } \\
\text { - Muestre cómo los valores se enlazan a } \\
\text { la estrategia de la empresa } \\
\text { - Haga visibles los intangibles. }\end{array}$ \\
\hline $\begin{array}{l}\text { Tomar mejores } \\
\text { decisiones basadas } \\
\text { en el valor }\end{array}$ & $\begin{array}{l}\text { - Priorizar: capacidades, } \\
\text { requisitos, funcionalidad } \\
\text { - Priorizar: mapas de rutas }\end{array}$ & $\begin{array}{l}\text { - Cree valores basados en la } \\
\text { priorización para proyectos, alcances, } \\
\text { requisitos, personalización, etc. }\end{array}$ \\
\hline $\begin{array}{l}\text { Mejorar la } \\
\text { rendición de } \\
\text { cuentas, la } \\
\text { alineación y el } \\
\text { seguimiento de } \\
\text { beneficios } \\
\end{array}$ & $\begin{array}{l}\text { - Asegurar la entrega de } \\
\text { valor } \\
\text { - Asignar la } \\
\text { responsabilidad del } \\
\text { seguimiento de resultados }\end{array}$ & $\begin{array}{l}\text { - Asigne a los responsables los } \\
\text { resultados de valor } \\
\text { - Haga seguimiento a los resultados de } \\
\text { valor } \\
\text { - Incruste prácticas de valor en el } \\
\text { programa de administración. }\end{array}$ \\
\hline General & $\begin{array}{l}\text { - Apoyar a la realización } \\
\text { del valor del ciclo de vida } \\
\text { del programa. } \\
\text { - Ampliar las discusiones } \\
\text { de los líderes de TI } \\
\end{array}$ & $\begin{array}{l}\text { - Aborde todas las fases del ciclo de } \\
\text { vida del programa } \\
\text { - Comunique con historias de valor. } \\
\text { - Informe y eduque a la alta gerencia } \\
\text { sobre el pensamiento basado en el valor. }\end{array}$ \\
\hline
\end{tabular}




\begin{tabular}{lll}
\hline Valor Necesario & Ejemplos de metas & Ejemplos de buenas prácticas \\
\hline & $\begin{array}{l}\text { centradas en el valor con los } \\
\text { negocios. } \\
\\
\\
\\
\text { orientación de valor. }\end{array}$ \\
\hline
\end{tabular}

Fuente: Elaboración propia basado en Keen (2011)

Maier y Remus (2003), crean conclusiones que pueden guiar a las organizaciones en la implementación de estrategias para la gestión del conocimiento orientada a procesos (PKM). Declaran tres actividades: la definición del punto de partida, definición de un anteproyecto y el cierre de brechas de enfoques. Para la segunda actividad sugiere implementar al principio del plan un proceso de negocio como QW, antes de que se implemente de manera incremental.

En cada proyecto piloto que se configure para asegurar QW, Watkins (2003) recomienda considerar responder a las siguientes interrogantes, para asegurarse de que se está estableciendo altos estándares para los tipos de comportamiento que se desea alentar.

- ¿Cuál es la combinación correcta de personas, en términos de conocimiento, habilidades y química personal?

- ¿Quién tiene la credibilidad, las habilidades de gestión del proyecto y la creatividad para liderar el proyecto?

- ¿Cuáles son los objetivos alcanzables de "estiramiento"?

- ¿Cuáles son los plazos alcanzables?

- ¿Qué entrenamiento o marco proporcionará para guiar la resolución de problemas y la toma de decisiones del equipo?

- ¿Qué otros recursos son necesarios para el éxito?

- ¿Cómo responsabilizará a las personas por lograr resultados superiores?

- ¿Cómo recompensará el éxito?

\section{Problemas de las QW}

Van Buren y Safferstone (2009), en su estudio plantean que más del 60\% de los líderes con bajo rendimiento han caído en al menos una de las cinco trampas que identificaron: centrándose demasiado en los detalles, reaccionando negativamente a la crítica, intimidando a los demás saltar a conclusiones y haciendo micro gestión.

Conway-King y Julien, (2018) señala que una QW es una inyección de adrenalina para una cultura de mejora continua. Las personas involucradas obtienen una gran satisfacción al hacer que el trabajo sea más efectivo, más eficiente o de menor costo; su esfuerzo da sus frutos y se amortiza rápidamente; están más inclinados a buscar otra mejora de este tipo. Las personas que ven o escuchan sobre el QW a menudo se inspiran para comenzar a buscar sus propios QW. Por lo tanto, el valor motivacional de una QW hace que el rendimiento del esfuerzo sea aún mayor. Pero ir tras QW no es una estrategia segura; sin un liderazgo efectivo, una organización puede terminar con "fallas rápidas" en su lugar; pueden darse situaciones a considerar como: 
- Para obtener una solución implementada rápidamente, un equipo puede omitir el análisis.

- A veces, cuando apunta a la velocidad, se apresura a juzgar, lo que resulta en una suboptimización.

- A menudo, una QW es solo una idea que alguien tiene "en el estante", es una idea que han estado llevando durante un tiempo.

- La velocidad no significa necesariamente que un equipo deba tomar atajos en la metodología de mejora de procesos.

\section{Conclusiones}

De los artículos evaluados el resultado de la presente revisión sistemática se ha llegado a concluir los siguientes puntos:

Springer fue la base de datos de publicaciones científicas que presentó más artículos relacionados con QW, el país desde el cual se realizaron la mayor cantidad de publicaciones fue en Estados Unidos y el 2017 fue el año de mayor presentación de resultados de investigaciones relacionadas con $\mathrm{QW}$.

La productividad, salud y TI fueron las áreas de aplicación a las que se han dirigido principalmente los estudios sobre QW; en menor cuantía ingeniería, emprendimiento, educación y medio ambiente. Las QW intervienen en procesos de mejora control, aumento de la productividad y la calidad, que a su vez impacta de manera próspera a la sociedad. En la salud, se han realizado importantes estudios en donde las QW han aportado favorablemente al cambio. Así mismo existe la aplicación de estrategias QW en los procesos y gestión de TI con buenos resultados.

Los líderes deben considerar en todo proceso las tareas relativamente cortas y simples relacionadas con los objetivos organizacionales; las cuales reducen la resistencia al cambio, aumentando la credibilidad e impulso de los colaboradores ante nuevos proyectos. Así mismo las perspectivas y habilidades para crear planes QW significativos, deben ser muy desarrolladas; existen alertas ya planteadas por experiencias, para no caer en las denominadas "fallas rápidas".

Finalmente, como investigación futura relacionada a las QW, se seleccionaría uno de los sectores evaluados, para estudiar el comportamiento local ante el uso de QW en sus actividades y proyectos.

\section{Referencias Bibliográficas}

Case, G. (2007). ITIL V3: Where To Start \& How To Achieve Quick Wins. Pink Elephant.

Chiaroni, D., Chiesa, V., Franzò, S., Frattini, F., \& Manfredi Latilla, V. (2017). Overcoming internal barriers to industrial energy efficiency through energy audit: A case study of a large manufacturing company in the home appliances industry. Clean Technologies and Environmental Policy, 19(4), 1031-1046. https://doi.org/10.1007/s10098-016-1298-5 
Conway-King, M. J., \& Julien, S. (2018). In Improvement as in many things, speed to results matters. Conway Management Company, Inc., 24, 4.

Corporate Executive Board. (2006). Making the Transition to First-Line Management. http://ceboard.vo.llnwd.net/o1/HRLC/IsManagementRightForMe/data/downloads/makin g_the_transition_to_first_line_management.pdf

Cristóbal, J., Castellani, V., Manfredi, S., \& Sala, S. (2018). Prioritizing and optimizing sustainable measures for food waste prevention and management. Waste Management, 72, 3-16. https://doi.org/10.1016/j.wasman.2017.11.007

Crouzet, B., W. Parker, D., \& Pathak, R. (2014). Preparing for productivity intervention initiatives. International Journal of Productivity and Performance Management, 63(7), 946-959. https://doi.org/10.1108/IJPPM-10-2013-0175

Desouza, K. C., Denford, J. S., \& Krishnamurthy, R. (2019). How the US Federal Communications Commission Managed the Process of IT Modernization. En N. Urbach \& M. Röglinger (Eds.), Digitalization Cases (pp. 411-428). Springer International Publishing. https://doi.org/10.1007/978-3-319-95273-4_22

Ferreras-Fernández, T., Martín-Rodero, H., García-Peñalvo, F. J., \& Merlo-Vega, J. A. (2016). The systematic review of literature in LIS: An approach. Proceedings of the Fourth International Conference on Technological Ecosystems for Enhancing Multiculturality TEEM '16, 291-296. https://doi.org/10.1145/3012430.3012531

Gal, D., \& McShane, B. B. (2012). Can Small Victories Help Win the War? American Marketing Association, 49, 487-501. https://doi.org/10.1509/jmr.11.0272

Gottsche, J., \& Kelly, M. (2018). Assessing the impact of construction waste reduction on selected projects in Ireland. Proceedings of the Institution of Civil Engineers - Waste and Resource Management, 171(3), 71-81. https://doi.org/10.1680/jwarm.17.00034

Gottsche, J., Kelly, M., \& Taggart, M. (2016). Assessing the impact of energy management initiatives on the energy usage during the construction phase of an educational building project in Ireland. Construction Management and Economics, 34(1), 46-60. https://doi.org/10.1080/01446193.2016.1162317

Grace, F. C., Meurk, C. S., Head, B. W., Hall, W. D., Harris, M. G., \& Whiteford, H. A. (2017). An analysis of policy success and failure in formal evaluations of Australia's national mental health strategy (1992-2012). BMC Health Services Research, 17(1), 374. https://doi.org/10.1186/s12913-017-2309-X

Grigg, N. P., Goodyer, J. E., \& Frater, T. G. (2018). Sustaining lean in SMEs: Key findings from a 10-year study involving New Zealand manufacturers. Total Quality Management \& Business Excellence, 1-14. https://doi.org/10.1080/14783363.2018.1436964

Guimarães, C. M., \& de Carvalho, J. C. (2012). Lean, a Tool Set or a Mind Set? A Healthcare Case Study. En H. Jodlbauer, J. Olhager, \& R. J. Schonberger (Eds.), Modelling Value (pp. 313-328). Physica-Verlag HD. https://doi.org/10.1007/978-3-7908-2747-7_16

Haque, A., \& Mantode, K. L. (2013). Governance in the Technology Era: Implications of Actor 
Network Theory for Social Empowerment in South Asia. En Y. K. Dwivedi, H. Z. Henriksen, D. Wastell, \& R. De' (Eds.), Grand Successes and Failures in IT. Public and Private Sectors (Vol. 402, pp. 375-390). Springer Berlin Heidelberg. https://doi.org/10.1007/978-3-642-38862-0_23

Houngbo, P. Th., Coleman, H. L. S., Zweekhorst, M., De Cock Buning, Tj., Medenou, D., \& Bunders, J. F. G. (2017). A Model for Good Governance of Healthcare Technology Management in the Public Sector: Learning from Evidence-Informed Policy Development and Implementation in Benin. PLOS ONE, 12(1), e0168842. https://doi.org/10.1371/journal.pone.0168842

Huang, S.-J., Wu, M.-S., \& Chen, L.-W. (2013). Critical success factors in aligning IT and business objectives: A Delphi study. Total Quality Management \& Business Excellence, 24(9-10), 1219-1240. https://doi.org/10.1080/14783363.2011.637785

Keen, J. M. (2011). Making Technology Investments Profitable. John Wiley \& Sons, Inc.

Maier, R., \& Remus, U. (2003). Implementing process-oriented knowledge management strategies. Journal of Knowledge Management, 7(4), 62-74. https://doi.org/10.1108/13673270310492958

Manterola, C., Astudillo, P., Arias, E., \& Claros, N. (2013). Revisiones sistemáticas de la literatura. Qué se debe saber acerca de ellas. Cirugía Española, 91(3), 149-155. https://doi.org/10.1016/j.ciresp.2011.07.009

Meyers, C. V., \& Hitt, D. H. (2018). Planning for school turnaround in the United States: An analysis of the quality of principal-developed quick wins. School Effectiveness and School Improvement, 29(3), 362-382. https://doi.org/10.1080/09243453.2018.1428202

Moshabela, M., Sene, M., Nanne, I., Tankoano, Y., Schaefer, J., Niang, O., \& Sachs, S. E. (2015). Early detection of maternal deaths in Senegal through household-based death notification integrating verbal and social autopsy: A community-level case study. BMC Health Services Research, 15(1), 16. https://doi.org/10.1186/s12913-014-0664-4

Rasmussen, N., Chen, C. Y., \& Bansal, M. (Eds.). (2015). Designing Metrics and Key Performance Indicators. En Business Dashboards (pp. 23-31). John Wiley \& Sons, Inc. https://doi.org/10.1002/9781119197904.ch5

Rixon, A., Maritz, A., \& Fisher, R. (2017). Youth Entrepreneurship in an Islamic Context. En V. Ramadani, L.-P. Dana, S. Gërguri-Rashiti, \& V. Ratten (Eds.), Entrepreneurship and Management in an Islamic Context (pp. 159-178). Springer International Publishing. https://doi.org/10.1007/978-3-319-39679-8_11

Rollin, P. F. (2012). 42 Rules for Your New Leadership Role (2nd Edition). SuperStarPress.

Sherman, R. (2013). Supply Chain Transformation. John Wiley \& Sons, Inc.

Surange, V. G. (2015). Implementation of Six Sigma to Reduce Cost of Quality: A Case Study of Automobile Sector. Journal of Failure Analysis and Prevention, 15(2), 282-294. https://doi.org/10.1007/s11668-015-9927-6 
Van Buren, M., \& Safferstone, T. (2009, enero). The Quick Wins Paradox. Harvard Business Review. https://hbr.org/2009/01/the-quick-wins-paradox

Warlé-van Herwaarden, M. F., Kramers, C., Sturkenboom, M. C., van den Bemt, P. M. L. A., \& De Smet, P. A. G. M. (2012). Targeting Outpatient Drug Safety: Recommendations of the Dutch HARM-Wrestling Task Force. Drug Safety, 35(3), 245-259. https://doi.org/10.2165/11596000-000000000-00000

Watkins, M. (2003). The first 90 days: Critical success strategies for new leaders at all levels. Harvard Business School Publishing.

Weber, S., \& Cooper, B. (2017). Moving slowly, not breaking enough: Trump's cybersecurity accomplishments. Bulletin of the Atomic Scientists, 73(6), 388-394. https://doi.org/10.1080/00963402.2017.1388676

Wray, J. (2016). Adapt to win: Creating value in an evolving business environment. EY-Parthenon. http://cdn.ey.com/parthenon/pdf/perspectives/Parthenon-EY_Adapt-toWin_ThoughtLeadership_Final_072016.pdf

Zevenbergen, C., van Herk, S., Escarameia, M., Gersonius, B., Serre, D., Walliman, N., de Bruijn, K. M., \& de Graaf, R. (2018). Assessing quick wins to protect critical urban infrastructure from floods: A case study in Bangkok, Thailand: Quick wins to protect critical urban infrastructure. Journal of Flood Risk Management, 11, S17-S27. https://doi.org/10.1111/jfr3.12173 